\title{
Electron Energy Loss Spectroscopy and Localized Cathodoluminescence Characterization of GaN Quantum Discs
}

\author{
Robert E.A. Williams ${ }^{1}$, Santino D. Carnevale ${ }^{2}$, Thomas. F. Kent ${ }^{1}$, David J. Stowe ${ }^{3}$, Roberto C. Myers ${ }^{1,2}$, \\ David W. McComb ${ }^{1}$ \\ ${ }^{1}$ Dept. of Materials Science and Engineering, The Ohio State University, Columbus, 43210 \\ 2 Dept. of Electrical and Computer Engineering, The Ohio State University, Columbus, 43210 \\ ${ }^{3}$ Gatan U.K. Abingdon, Oxon., United Kingdom
}

Nanomaterials with unique optical and electronic properties are important for ongoing developments in optoelectronics, telecommunication devices and sensor technology. Critical to the development and application of such nanomaterials is the ability to locally measure the electronic band gap in order to evaluate quantum well behavior and device. Traditionally, band gaps are characterized using optical methods that have high spectral resolution $(<2 \mathrm{meV})$ but poor spatial resolution $(\sim 10 \mu \mathrm{m})$. [1] These resolution limits are not sufficient for rigorous analytical characterization of modern devices based on nanostructured materials. Electron energy-loss spectroscopy (EELS) performed in the scanning transmission electron microscope (STEM) has the potential to probe the band gap regime with nanometer-scale spatial resolution, and if this could be combined with simultaneous determination of the luminescent properties it would provide a key technique to probe novel optoelectronic systems. [2,3] Zagonel et. al. recently demonstrated the feasibility of this concept using a custom-made cathodoluminescence system. [4]

This work combines HAADF-STEM imaging, valence EELS and localized cathodoluminescence (CL) in an aberration corrected, monochromated FEI Titan 80-300 equipped with a Gatan Vulcan system. [4] Implementing this experimental setup allowed for correlated mapping of structure, bandgap and optical luminescence signals. Collection of valence EELS spectra enables band gap measurement as a function of position, providing experimental investigation of the electronic structure. For semiconductors, Cherenkov losses must be taken into account, an iterative procedure can be applied to remove Cherenkov radiation and allow band gap measurement directly. To minimize the Cherenkov contribution, spectra were collected at $80 \mathrm{kV}$. Furthermore, the monochromator was excited to provide $\sim 0.2 \mathrm{eV}$ spectral resolution with a symmetric zero-loss profile. CL measurements, which consist of excitation position resolved emission, allowed for direct probing of exciton recombination sites within the nanowire heterostructure. Comparison of CL spectral maps with EELS bandgap maps allows for interpretation of likely recombination sites and optical characteristics in terms of an individual nanowire's experimental vs theoretical band-diagram. Finally, the correlation of CL spectra with position from the HAADF STEM image allows identification of optically dominant excitation sites within an individual nanowire quantum disk. The data to be presented was collected from self-assembled GaN III-Nitride nanowire heterostructures.

Figure 1 shows a HAADF image and representative spectra extracted from various regions of the nanowire. Additionally, the CL map enabled pixel averaging and produced better signal to noise for given regions of the nanowire. The spectra show that the CL becomes bright and narrow at 380nm for excitation in the center of the quantum disk, conversely, for excitations away from the quantum disk, CL becomes more broad and less intense. Figure 2 (a) shows a plot of wavelength vs. intensity and a sharp reduction in $\mathrm{CL}$ is observed for excitations just to side of the quantum disk. 
References:

[1] L. Gu, et al, Physical Review B 75, (2007)

[2] P. Batson, et al, Ultramicroscopy 22, (1987)

[3] R. Erni, et al, Ultramicroscopy, (2005)

[4] Zagonel, et al, Nanoletters 11, (2010)

[4] D. Stowe, Microscopy and Microanalysis 19, (2013)

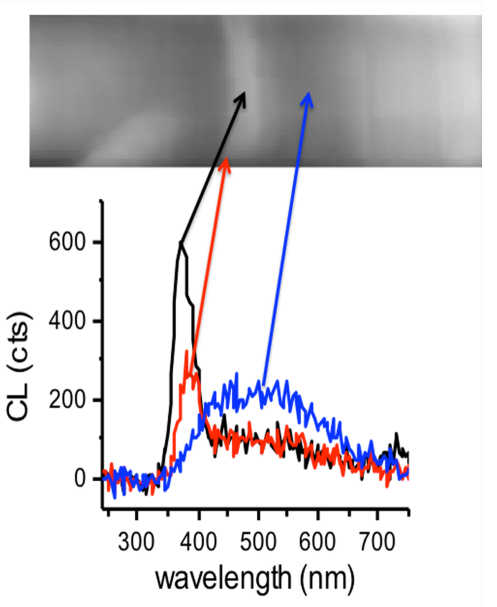

Figure 1: (a) HAADF STEM image showing line scan across $2 \mathrm{~nm}$ GaN quantum well. (b) CL excitation for middle, edge and off of the quantum well.
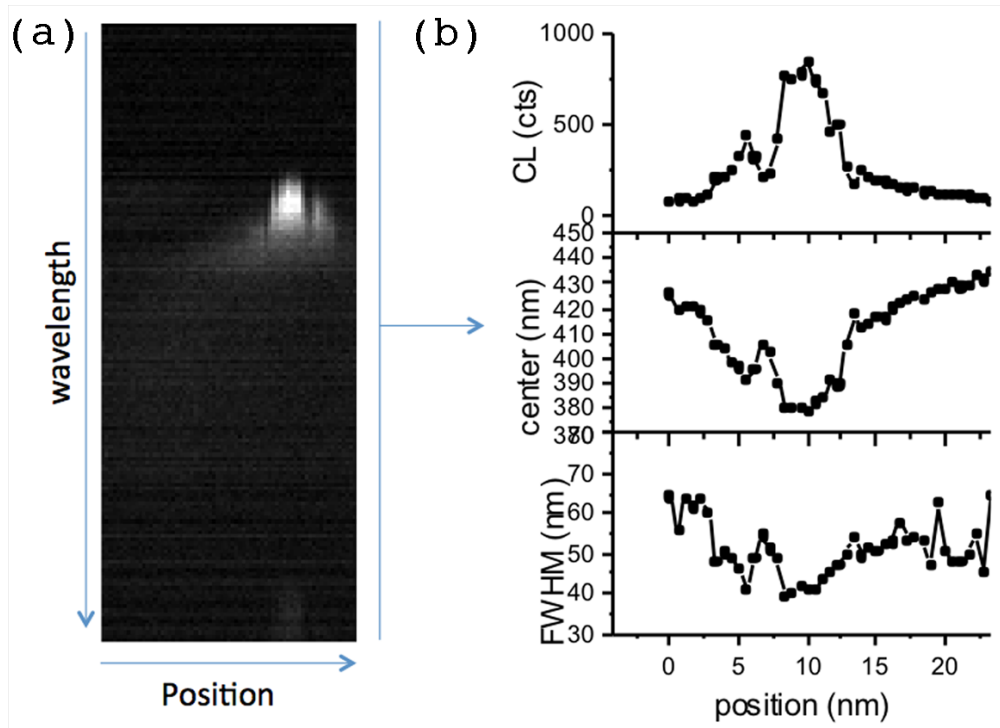

Figure 2: (a) 2D spectral map showing wavelength vs. position across a $2 \mathrm{~nm}$ GaN quantum well. (b) CL excitation showing a sharp reduction in excitation to the side of the quantum well. 